\title{
Formation of Three Dimensional Ni Nanostructures for Large Area Catalysts
}

\author{
J. D. Carey, S. J. Henley, E. Mendoza, C. E. Giusca, A. A. D. T. Adikaari and S. R. P. Silva \\ Advanced Technology Institute, School of Electronics and Physical Sciences, \\ University of Surrey, Guildford, GU2 7XH.
}

\begin{abstract}
The formation of Ni nanostructures to act as catalysts in the growth of carbon nanotubes is reported. The changes in the surface morphology of Ni produced by three methods - thermal evaporation and annealing of thin films, pulsed laser ablation and annealing of $\mathrm{Ni}$, and the use of metal containing macromolecules - have been investigated by atomic force microscopy and scanning electron microscopy. In the case of thermal annealing of thin metal films in the temperature range $300-500^{\circ} \mathrm{C}$ we observe an increase in the mean diameter of the islands formed, accompanied by a reduction in the mean island density with increasing temperature. We attribute this effect to mass transport of weakly bound individual $\mathrm{Ni}$ atoms and/or small island clusters across the surface to form larger isolated islands, in a process similar to Ostwald ripening. Using a pulsed $\mathrm{KrF}$ excimer laser for ablation of a Ni target we show that nanometre smooth Ni thin films can be produced provided a sufficient number of laser shots is used. The surface morphology of these smooth films can then be altered by laser annealing to form $\mathrm{Ni}$ droplets. It is found that the mean diameter of the $\mathrm{Ni}$ droplets depends not only on the initial $\mathrm{Ni}$ thickness but also the laser fluence. It is also found that the nanostructuring of the film depends on the presence of an oxide under layer, with a higher fluence required on thinner oxides and no nanostructuring observed on bare Si. Finally, we show that Ni nanostructuring can be formed by suitable annealing of a $\mathrm{Ni}$ containing aqueous dendrimer solutions.
\end{abstract}

\section{INTRODUCTION}

Future applications in large area electronics may, ironically, be driven by a greater understanding of material systems at a nanometre level. To that end, the preparation of large area substrates, ideally at low temperatures $\left(<600{ }^{\circ} \mathrm{C}\right)$, that can be used either directly or as catalysts for subsequent growth is extremely important. Carbon nanotubes (CNTs) is one material system where an ability to grow over large areas may lead to significant developments towards the next generation of wide screen field emission based flat panel displays [1]. The controllable growth of CNTs is, however, often determined by properties such as mean catalyst diameter, distribution of the catalyst across the substrate and the mutual interaction between the growing nanotube, the catalyst and the substrate itself [2]. This is in addition to the changes in process parameters such as type and flow rates of source gases, growth temperature and pressure. The latter quantities are readily determined by the user, and as a result an ability to predetermine the catalyst properties before growth is important. This proposition holds true for both conventional large area catalysts and also for catalysts defined lithographically. In this study we use thin Ni metal and three different techniques to alter its morphology - thermal annealing of thin films, pulsed laser ablation to produce the film and then laser annealing to alter the film and an aqueous macromolecular solution than can be spin coated. 


\section{EXPERIMENTAL METHOD}

For the annealing of thin metal films, substrates of $n$-Si were initially cleaned using a standard three stage ultrasonic solvent clean. A layer of Ti $100 \mathrm{~nm}$ thick was evaporated on the substrates by heating Ti metal in a vacuum of $4 \times 10^{-6}$ Torr. The films were then subsequently oxidized in air by annealing for 30 minutes. $\mathrm{Ni}$ films were then evaporated using high purity $\mathrm{Ni}$ wire which were subsequently annealed under flowing $\mathrm{N}_{2}$ at temperatures from $300^{\circ} \mathrm{C}$ up to $500^{\circ} \mathrm{C}$ for times of 10,30 and 60 minutes.

For pulsed laser ablation studies, thin Ni films were produced by multiple laser shots (102000) of a Lambda-Physik LPX 200 Excimer laser operating at $248 \mathrm{~nm}$ at a fluence of $10 \mathrm{~J} / \mathrm{cm}^{2}$ onto a $99.9 \%$ pure Ni target. Further details can be will be published elsewhere [3]. The substrates used were $\mathrm{SiO}_{2} / \mathrm{Si}$ with either native oxide coverage or with a thermal oxide of $235 \mathrm{~nm}$ or $320 \mathrm{~nm}$ thickness. Laser annealing of the resultant thin films was performed using the same excimer laser as for growth. The samples were attached to a translation stage and annealing in vacuum was performed by translating the stage at $1 \mathrm{~mm} / \mathrm{s}$ with a laser repetition rate of $10 \mathrm{~Hz}$. For the annealing studies, laser fluences from 100 to $300 \mathrm{~mJ} / \mathrm{cm}^{2}$ were used.

Finally, dendrimers, which are metal containing macromolecules, were prepared from commercially [4] available polyamidoamine $6^{\text {th }}$ Generation dendrimer solution with $100 \% \mathrm{OH}$ terminated surface groups. Using $48 \mu \mathrm{L}$ of $17.83 \%$ wt. of dendrimer mixed with $100 \mu \mathrm{L}$ of 0.1 $\mathrm{M} \mathrm{NiCl}_{3} \cdot 6 \mathrm{H}_{2} \mathrm{O}$, a $10 \mathrm{ml}$ solution was obtained using double distilled water. A low concentration was formed by diluting the stock solution 100 times. Silicon substrates with native oxide were soaked in the diluted dendrimer solution for 5 seconds, rinsed with double distilled water to remove poorly attached or polymerised dendrimers from the substrate and blown dry with nitrogen. The substrates were annealed in a vacuum of $10^{-3}$ Torr at $650{ }^{\circ} \mathrm{C}$ for 5 minutes.

The surface morphology of all the substrates were examined using atomic force microscopy (either a Digital Instruments Nanoscope IIIa or a Dimension 3100 microscope). Scanning electron microscopy using a Hitachi S4000 field emission gun scanning electron microscope (SEM) was also employed.

\section{RESULTS AND DISCUSSION}

\section{Thermal annealing of thin metal films}

High resolution AFM measurements (not presented here) of the unannealed Ni films with a thickness of $8 \mathrm{~nm}$ show that the surface of the film to be extremely flat. However, the surface morphology changes upon annealing for 30 minutes as shown in Fig. 1. After annealing at 300 ${ }^{\circ} \mathrm{C}$, modulations of the surface can be see with evidence of small localised islands present. Annealing at $400^{\circ} \mathrm{C}$ (Fig. 1 (b)) produced larger islands with an irregular shape and a mean diameter of $114 \mathrm{~nm}$. The increase in the mean size of the islands is coupled with an increase in the larger inter-island spacing. This increase in the mean size and separation is further seen after annealing at $500^{\circ} \mathrm{C}$. In this case, large diameter islands (average diameter of $306 \mathrm{~nm}$ and a standard deviation of $97 \mathrm{~nm}$ ) can be seen in Fig. 1(c), and the resultant islands are also more isolated from each other. The results shown in Fig. 1 demonstrate that annealing at successively higher temperatures results in the formation of isolated large diameter islands at the expense of smaller close packed islands. 

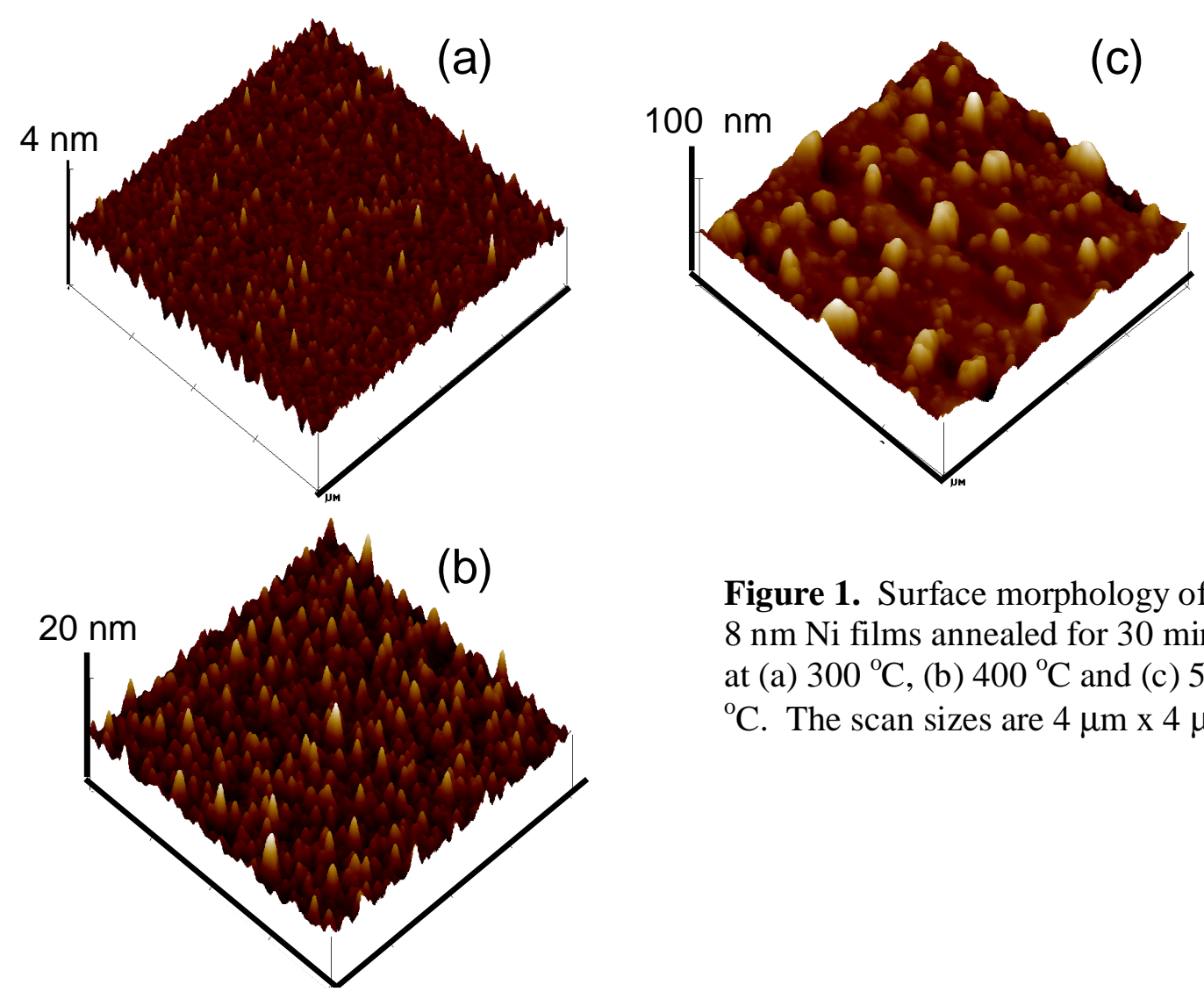

Figure 1. Surface morphology of three $8 \mathrm{~nm}$ Ni films annealed for 30 minutes at (a) $300{ }^{\circ} \mathrm{C}$, (b) $400{ }^{\circ} \mathrm{C}$ and (c) 500 ${ }^{\circ} \mathrm{C}$. The scan sizes are $4 \mu \mathrm{m} \times 4 \mu \mathrm{m}$.

Although the deposition of $\mathrm{Ni}$ results in compressive strain on the oxidised titanium, the strain energy is not substantially reduced through thermal annealing since the coefficients of linear expansion [5] for $\mathrm{Ni}$ and the oxide, taken to be $13.4 \times 10^{-6} \mathrm{~K}^{-1}$ and $7.14 \times 10^{-6} \mathrm{~K}^{-1}$ respectively, are similar. The effect of annealing at high temperatures is to allow mass transport of either individual $\mathrm{Ni}$ atoms or small clusters across the surface in which larger clusters can be formed out of smaller clusters in a process analogous to Ostwald ripening. Such a process is consistent with the low interaction of $\mathrm{Ni}$ atoms on oxide substrates. Metals which possess high heats of formation of the oxide react strongly with the substrate and produce flat 'wetting layers'. The heats of formation of the oxide, $-\Delta \mathrm{H}$, are $\sim 5.2 \mathrm{eV}$ for Ti and $2.49 \mathrm{eV}$ for Ni [6]. Since the value of $-\Delta \mathrm{H}$ for $\mathrm{Ni}$ is about half that of $\mathrm{Ti}$, the growth of $\mathrm{Ni}$ will result in little interaction with the substrate. As a result low barriers of atom diffusion on the surface are possible. Further confirmation of a surface diffusion hypothesis leading to larger islands can be found from the examination of the island density inferred for the AFM measurements. On thermal annealing, the density of islands decreases from $32.6 \mu \mathrm{m}^{-2}$ at $300^{\circ} \mathrm{C}$, to $15.6 \mu \mathrm{m}^{-2}$ at $400^{\circ} \mathrm{C}$ and to only 2.3 $\mu \mathrm{m}^{-2}$ after annealing at $500^{\circ} \mathrm{C}$.

For thinner metal layers of $3 \mathrm{~nm}$ and $5 \mathrm{~nm}$ it was found that after annealing at $400{ }^{\circ} \mathrm{C}$ for 30 minutes island formation has occurred but with mean diameters of $67 \mathrm{~nm}$ and $89 \mathrm{~nm}$ respectively. These values can be compared to $114 \mathrm{~nm}$ found for $8 \mathrm{~nm}$ thick. Annealing studies at 10 and 60 minutes produced similar results from which we conclude that the two most important aspects in producing $\mathrm{Ni}$ islands are the annealing temperature and the initial $\mathrm{Ni}$ layer 
thickness. Annealing at higher temperatures produces larger diameter islands though further in distance from one another. The weak interaction between the $\mathrm{Ni}$ and the oxide layer results in a low activation energy barrier to Ni migration which results in larger islands forming from smaller islands. In this way the kinetic factors which affect mass transport and surface diffusion of material are an important consideration in addition to simple thermodynamic factors since metal-on-oxide often represents a far from equilibrium system [7].

\section{Laser ablation, annealing and droplet formation of Ni nanostructures}

The initial stages of growth of the Ni films by laser ablation onto a $320 \mathrm{~nm}$ thick $\mathrm{SiO}_{2}$ on $\mathrm{Si}$ substrate was investigated using AFM. Figure 2 shows the evolution of the surface after 10, 50 and 200 laser shots at a fluence of $10 \mathrm{~J} / \mathrm{cm}^{2}$. The scan sizes are $400 \mathrm{~nm}$ x $400 \mathrm{~nm}$ in each case.
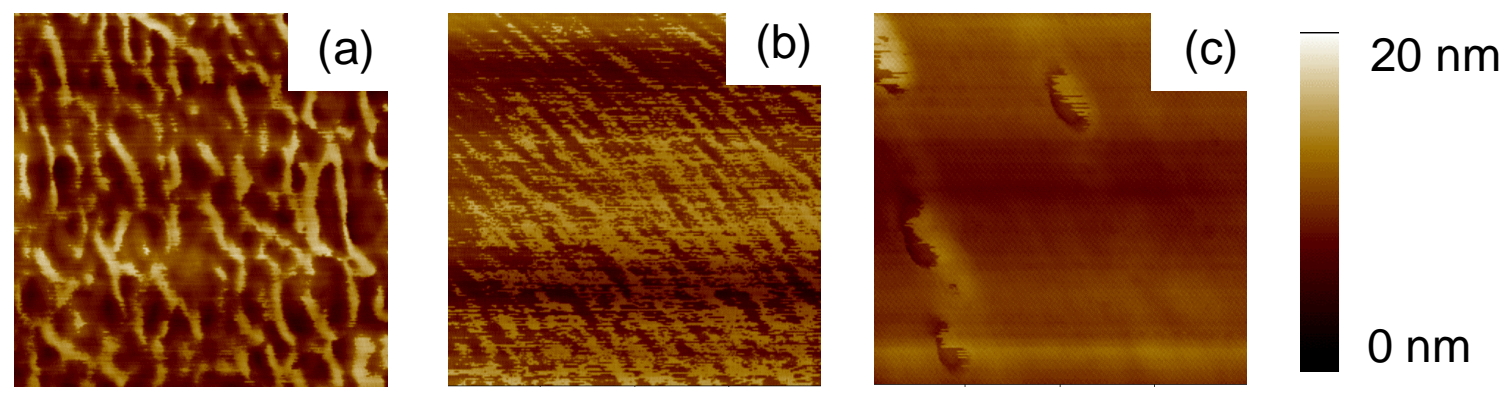

Figure 2. AFM images of laser ablated Ni for (a) 10 (b) 50 and (c) 200 laser shots at $10 \mathrm{~J} / \mathrm{cm}^{2}$.

For a low number of laser shots (10 shots), the surface is not continuous, however, film continuity begins to emerge at around 200 shots and by 1000 shots a smooth film with a root mean square roughness of less than $1 \mathrm{~nm}$ emerged. By masking a section of the substrate prior to deposition, it was possible to measure the Ni film thickness by scanning the AFM over the step produced. 750 laser shots produced a film $6.5 \mathrm{~nm}$ thick and 1500 shots resulted in a thickness of $8.2 \mathrm{~nm}$.

Figure 3 shows series of SEM images of a selection of the laser annealed films, with different initial $\mathrm{Ni}$ thickness, grown on $320 \mathrm{~nm} \mathrm{SiO} / / \mathrm{Si}$ substrates annealed with a laser fluence of 160 $\mathrm{mJ} / \mathrm{cm}^{2}$. After annealing the Ni film was observed to break up into discrete nanometer-scale hemispherical islands - metal droplets.
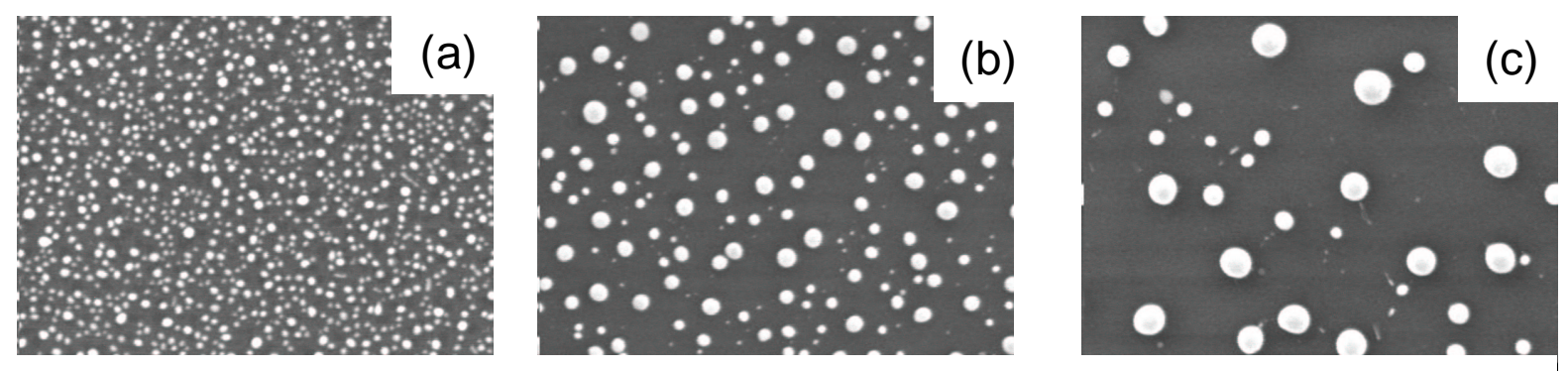

Scale Bar $300 \mathrm{~nm}$

Figure $3 \mathrm{SEM}$ images of three films with thickness (a) $6.5 \mathrm{~nm}$ (b) $8.2 \mathrm{~nm}$ and (c) $12 \mathrm{~nm}$ annealed at a laser fluence of $160 \mathrm{~mJ} / \mathrm{cm}^{2}$. The error in film thickness is $\pm 1 \mathrm{~nm}$. 
At fluences below a critical value (which was dependent on the Ni thickness), the films were observed to perforate, but droplet formation did not occur. For fluences above $280 \mathrm{~mJ} / \mathrm{cm}^{2}$ significant ablation of the $\mathrm{Ni}$ was observed for all $\mathrm{Ni}$ film thicknesses investigated. Whilst it was observed that at intermediate fluences the Ni island size was unaffected by the laser fluence, the fluence required to achieve droplet formation does depend on the thickness of the oxide layer on the substrate. The fluence required to nanostructure the films grown on the thinner $(235 \mathrm{~nm})$ thermal $\mathrm{SiO}_{2}$ substrates was higher than for the corresponding $\mathrm{Ni}$ film on the thicker $\mathrm{SiO}_{2}$ layers.

On the Si substrates with only the native oxide layer, no nanostructuring was observed for fluences below the ablation threshold of the film. This attributed to the higher thermal conductivity of $\mathrm{Si}\left(150 \mathrm{Wm}^{-1} \mathrm{~K}^{-1}\right)$ when compared with that of $\mathrm{SiO}_{2}\left(1.34 \mathrm{Wm}^{-1} \mathrm{~K}^{-1}\right)$. [5] It can be observed that the mean droplet size increases as the initial film thickness increases, which is consistent with the thermal annealing data of thin films, shown in Fig. 1. However, unlike the Ni island formed by thermal annealing the Ni droplets formed here are prepared without any macroscopic heating of the substrate.

\section{Ni nanostructures from dendrimer macromolecules}

Figure 4(a) shows an AFM image of the surface of Si substrate on to which dendrimers have been adsorbed by soaking in the diluted solution and subsequent drying. The surface consists of circular features with a diameter of few tens of nanometres. After thermal annealing at $650^{\circ} \mathrm{C}$ for 5 mins evidence of coalescence of the dendrimers into larger features is observed, Fig. 4(b).
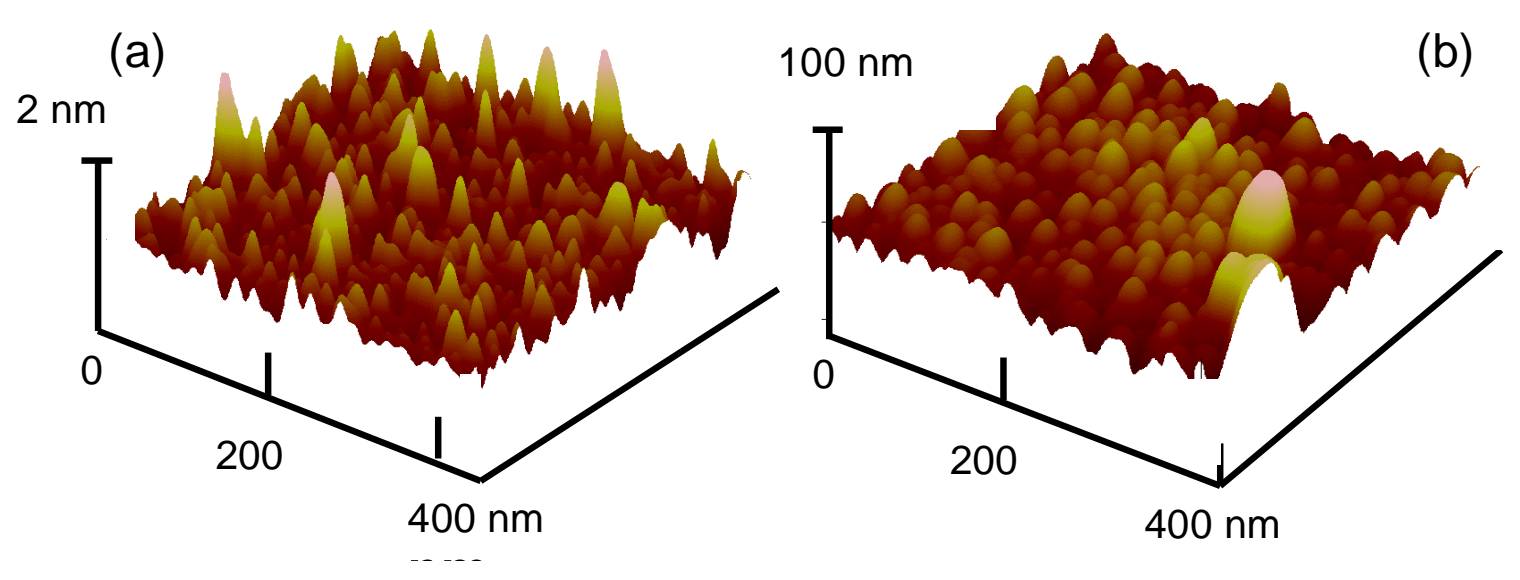

Figure 4 AFM image of (a) the dried as-grown dendrimer and (b) annealed at $650{ }^{\circ} \mathrm{C}$ for 5 minutes.

By using a combination of high resolution transmission electron microscopy and energy dispersive X-ray analysis it has been shown that the nanostructure also consists of Ni particles surrounded by a thin $(3-5 \mathrm{~nm})$ carbon shell. The presence of the $\mathrm{C}$ shell surrounding the $\mathrm{Ni}$ has a benefit over a bare Ni surface since the carbon acts as a barrier to diffusion of the metal atom into the silicon substrate, at high temperatures, and inhibits the formation of a silicide. This results in the growth of mats of CNT over large areas [8]. 


\section{CONCLUSIONS}

In this paper we have demonstrated the formation of Ni nanostructures using conventional thermal annealing, laser annealing, and annealing of metal containing macromolecules. For a given metal thickness, conventional thermal annealing results in the formation of a smaller number of larger diameter metal islands. The thermal energy supplied to the whole of the film results in the transport of smaller islands to form larger islands. Smooth Ni thin films can be produced from laser ablation of a Ni target which can then be nanostructured by pulsed laser annealing. The laser fluence needed to form Ni droplets depends on the thermal conductivity of the underlying substrate, and higher fluences were required on thinner oxides with no nanostructuring also observed on bare Si. Finally, we have demonstrated that annealing of Ni dendrimers can result in Ni nanostructures. This final approach allows the use of an aqueous solution than is compatible with spin coating technology and may provide a way to produce large area catalysed substrates.

\section{ACKNOWLEDGEMENTS}

The authors would like to acknowledge the EPRSC for funding under the Portfolio Partnership and the Carbon Based Electronics research program. JDC acknowledges the EPSRC for funding via an Advanced Research Fellowship. EM acknowledges the Generalitat de Catalunya for the Nanotec Postdoctoral Fellowship Program

\section{REFERENCES}

1. K.A. Dean, B.R. Chalamala, B.F. Coll, Y.W.C. Xie and J.E. Jaskie, New Diam. Frontier Carbon Tech. 12, 165 (2002).

2. V. I. Merkulov, D.H. Lowndes, Y. Wei, G. Eres and E. Voelkl, Appl. Phys. Lett. 76, 3555 (2000).

3. S. J. Henley, C. H. P. Poa, A. A. D. T Adikaari, C. E. Giusca, J. D. Carey and S. R. P. Silva, accepted for publication in Applied Physics Letters.

4. See, for example, www.dendritech.com

5. G. W. C. Kaye and T. H. Laby in Tables of physical and chemical constants, Longman, London, UK, 15th edition, 1993.

6. U. Diebold, J.-M. Pan and T.E. Madey, Phys. Rev. B. 47, 3868 (1993).

7. J. D. Carey, L. L. Ong, and S. R. P. Silva, Nanotechnology 14, 1223 (2003).

8. E. Mendoza, C. H. P. Poa, S. J. Henley, V. Stolojan, G. Y. Chen, C. E. Giusca, J. D. Carey and S. R. P. Silva, unpublished. 